\title{
Large Thyroid Cysts
}

Ancuta Augustina Gheorghisan-Galateanu ${ }^{1}$, Mara Carsote ${ }^{1}$, Claudiu Tupea ${ }^{2}$,

Anda Dumitrascu ${ }^{2}$, Ana Valea ${ }^{3}$, Eugenia Petrova ${ }^{2}$, Andrei Goldstein ${ }^{2}$, Adina Ghemigian ${ }^{1}$

1.C.Davila University of Medicine and Pharmacy \& C.I.Parhon National Institute of Endocrinology, Bucharest, Romania

2.C.I.Parhon National Institute of Endocrinology, Bucharest, Romania

3.I. Hatieganu University of Medicine and Pharmacy \& Clinical County Hospital, Cluj-Napoca, Romani

\section{Introduction}

Thyroid fluid-filled cavities are mostly resulted from degenerating thyroid adenomas but blood vessel anomalies are also described. Despite the typical benign behavior the local anatomical effects may require fine needle aspiration or directly surgery.

\section{Material \& Methods}

This is series of cases presenting intermittent breathing problems leading to the diagnosis of thyroid cysts. Thyroid evaluation included ultrasound, computed tomography (CT), TSH assay (Thyroid Stimulating Hormone), TPO antibodies (anti-thyreoperoxidase), circulating calcitonin.

CT scan: large cervical cyst on a 47 year old female

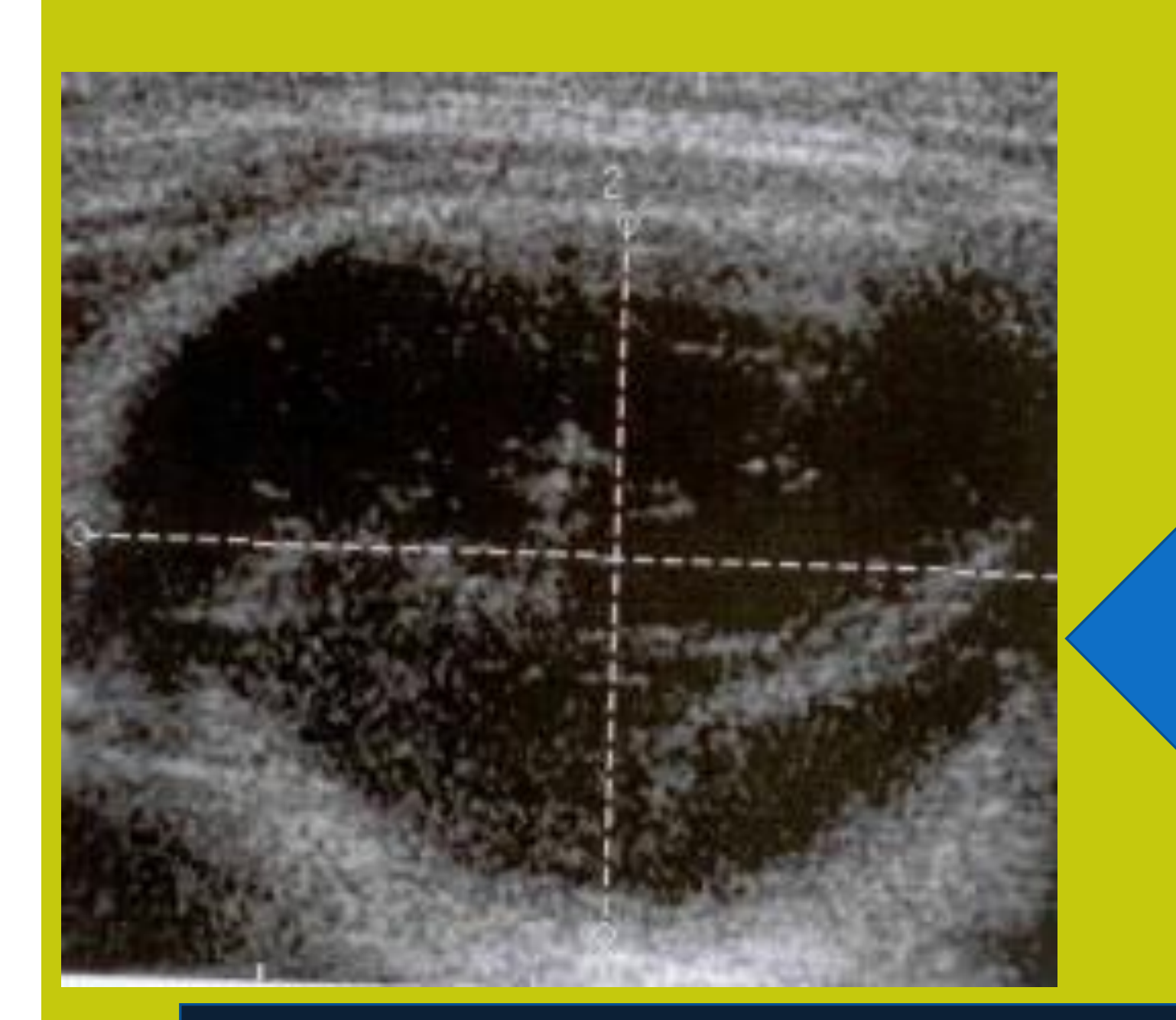

Neck ultrasound: large thyroid cyst on a 28-year old female
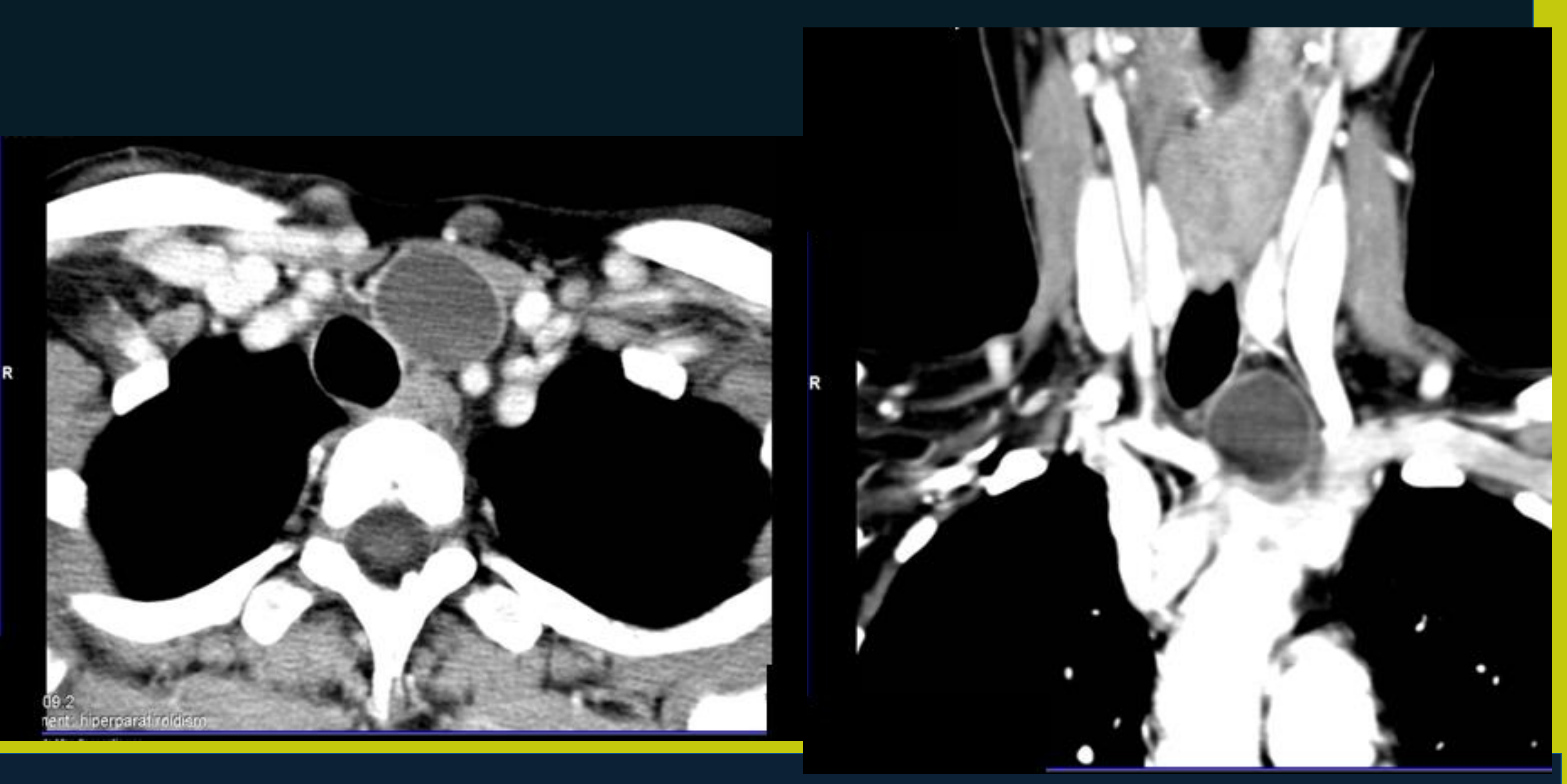

Results

A 28-year female was evaluated for irregular menses, weight gain, and occasional dyspnea. The personal medical history is irrelevant.

The investigations confirmed polycystic ovaries syndrome but also a hemorrhagic thyroid cyst of 4.3 by 2.9 by $2.88 \mathrm{~cm}$ (with several hyper-echoic areas insight, and a left deviation of the trachea) was detected by ultrasound (a right thyroid lobe of 3.15 by 3.3 by $5.35 \mathrm{~cm}$, left lobe of 1.92 by 1.53 by $4.82 \mathrm{~cm}$ ) with homogenous structure. Thyroid profile was normal: TSH=0.78 $\mu \mathrm{UI} / \mathrm{mL}(\mathrm{N}: 0.5-4.5 \mu \mathrm{UI} / \mathrm{mL}), \mathrm{TPO}=10 \mathrm{UI} / \mathrm{mL}(\mathrm{N}: 0-$ $35 \mathrm{UI} / \mathrm{mL}$ ), FreeT4=14.3pmol/L (N:10.3-24.4 pmol/L), calcitonin=3.38pg/mL (N:5.17-9.82 pg/mL). Total thyroidectomy is recommended.

A 47-year female, known with Raynaud's syndrome and euthyroid Hasmimoto's thyroiditis presents from the last two weeks mild breathing troubles. 15 years ago she has an episode of nephritic syndrome and corticotherapy was offered for a few months. On admission, thyroid profiles reveled: TSH=1.19 $\mu \mathrm{UI} / \mathrm{mL}$, $\mathrm{TPO}=143 \mathrm{UI} / \mathrm{mL}$, calcitonin $=1 \mathrm{pg} / \mathrm{mL}$ with a hypo-echoic ultrasound pattern and a large cyst of 3 by 2.32 by $2.93 \mathrm{~cm}$ with negative 131 iodine uptake at scintigram.

CT pointed a well shaped, encapsulated cyst emerging to the anterior superior mediastinum of 17-33 Hounsfield Units. The mass removal was done and benign features were found.

\section{Conclusion}

Regarding the first case, the polycystic ovaries syndrome is most probably incidental with the thyroid cystic lesion. Whether autoimmune background and previous prednisone administration may contribute to a thyroid cyst formation is difficult to establish. 